SHORT REPORT

\title{
Paradoxical absence of nuclear inclusion in cerebellar Purkinje cells of hereditary ataxias linked to CAG expansion
}

\author{
S Koyano, K Iwabuchi, S Yagishita, Y Kuroiwa, T Uchihara
}

J Neurol Neurosurg Psychiatry 2002;73:450-452

\begin{abstract}
Degeneration of cerebellar cortex is one of the principal features of hereditary ataxias linked to expansion of CAG repeat. In an attempt to clarify possible correlation between neuronal depletion and neuronal intranuclear inclusions, both triggered by the pathological expansion of CAG repeat, cerebellar sections from SCA1, SCA2, SCA3, and DRPLA cases were immunostained with anti-ubiquitin or anti-expanded polyglutamine antibody (1C2) and were screened for the presence of neuronal intranuclear inclusions. Although the degree of cerebellar degeneration varied greatly, cerebellar Purkinje cells were uniformly characterised by the absence of neuronal intranuclear inclusion. Complete absence of neuronal intranuclear inclusion in Purkinje cells is apparently paradoxical and hardly explained if neuronal intranuclear inclusion formation is positively correlated to a mechanism accelerating neuronal death. It may, otherwise, suggest an intrinsic link between neuronal intranuclear inclusion formation and neurodegeneration in opposite directions in human Purkinje cells, more or less affected in these CAG repeat disorders.
\end{abstract}

$A^{\prime}$ least 10 inherited neurodegenerative diseases, including spinocerebellar ataxias (SCA-1, -2, -3, -6, -7, -12, and -17), Huntington's disease, dentatorubral pallidoluysian atrophy (DRPLA), and spinobulbar muscular atrophy (SBMA) are associated with pathologically expanded CAG repeats in the coding region of the disease gene..$^{1-3}$ Because most of them are characterised by the presence of neuronal intranculear inclusions (NIs), which contain the gene product derived from the expanded allele of the responsible gene, formation of NIs induced by expansion of CAG repeat may play a pivotal part in the pathogenesis in common. Although it has been established that an artificial expression of expanded CAG repeat in cultured cells or in transgenic animals induces formation of NIs, it remains to be clarified how NIs are involved in the pathogenesis and neurodegeneration. Accumulating evidence suggests, however, that NI formation is not necessarily correlated with the severity of neuronal degeneration. Discrepancy between neuronal degeneration and formation of NIs has been observed at necopsy in brains with Huntington's disease $^{4}$ or with neuronal intranuclear hyaline inclusion disease (NIHID), ${ }^{5}$ in mouse transgenic lines carrying mutated ataxin- $1,{ }^{6}$ and in some cellular models of polyglutamine diseases. ${ }^{7}$ Because it is essential to know how NIs are involved in these degenerative processes, we systematically screened cerebellar cortex in a series of hereditary ataxias in search of ubiquitin or polyglutamine-immunopositive NIs, with special attention to cerebellar Purkinje (Pj) cells, more or less affected in these disorders. A possible role of NI in neurodegeneration and its relation to neuronal death will be discussed.

\section{METHODS}

Sections were obtained at postmortem examination from the brains of three SCA1, three SCA2, six SCA3, and five DRPLA patients from unrelated families and three control subjects without neurological and psychiatric disorders. Diagnoses were based on clinical and pathological findings and were confirmed by genomic DNA analysis, as reported previously. ${ }^{8}$ Each cerebellum was fixed in $10 \%$ formalin for one to three weeks, then cut into $1 \mathrm{~cm}$ thick sagittal slices for histopathological and immunohistochemical examination. They were embedded in paraffin wax and four serial slices of $5 \mu \mathrm{m}$ thick sections were immunostained with an anti-ubiquitin antibody ( $1: 1000$, rabbit polyclonal IgG, DAKO, Glostrup, Denmark) or with an anti-polyglutamine antibody, 1C2 (1:9000, mouse monoclonal IgG, Chemicon, Temecula, CA) by using the avidin-biotin-peroxidase complex method (ABC Elite, Vector Burlingane, CA).

\section{RESULTS}

Pathological features of these cases were previously summarised. ${ }^{8}$ In SCAl brains, mild to moderate degeneration of the pontocerebellipetal system and of the Pj cells of the cerebellar cortex was found and neuronal loss with grumose degeneration was consistent in the dentate nucleus. SCA2 brains were characterised by severe gliosis involving all three layers of the cerebellar cortex, and loss of $\mathrm{Pj}$ cells was uniformly severe in all cases. The dentate nucleus was spared, while some fibrillary gliosis may be seen there in SCA2 brains. In SCA3 and DRPLA brains, the cerebellar cortex was mostly spared, but a slight loss of Pj cells was recognisable. Grumose degeneration was remarkable in the dentate nucleus.

The anti-ubiquitin antibody, at most, lightly immunostained the nucleoplasm and to a lesser extent the cytoplasm of $\mathrm{Pj}$ cells in all cases (fig l A-D). Ubiquitin-immunopositive NIs were occasionally found in the dentate nucleus of SCAl, SCA3 (fig l E) and DRPLA (fig l F) cases and rarely in Golgi cells in SCAl (fig l G), SCA2, and DRPLA cases. These NIs were also immunostained with the antibody against expanded polyglutamine (1C2), as shown in fig $1 \mathrm{H}$ (Golgi cell in SCAl). These two antibodies yielded no significant immunolabelling in control cases. Concerning Pj cells, we counted the number of Pj cells in each four serial slices of cerebellum in every case examined in this study (table 1). Approximate mean number of remaining $\mathrm{Pj}$ cells per section traversing the dentate nucleus, each including whole sagittal plane of a hemisphere, was about 420 in SCA1, 150 in SCA2, 2320 in SCA3, and 3130 in DRPLA. Neither anti-ubiquitin antibody nor 1C2 identified

Abbreviations: $\mathrm{NI}$, neuronal intranuclear inclusion; $\mathrm{P}$, Purkinje; DRPLA, dentatorubal pallidoluysian atrophy; SBMA, spinobulbar muscular atrophy 

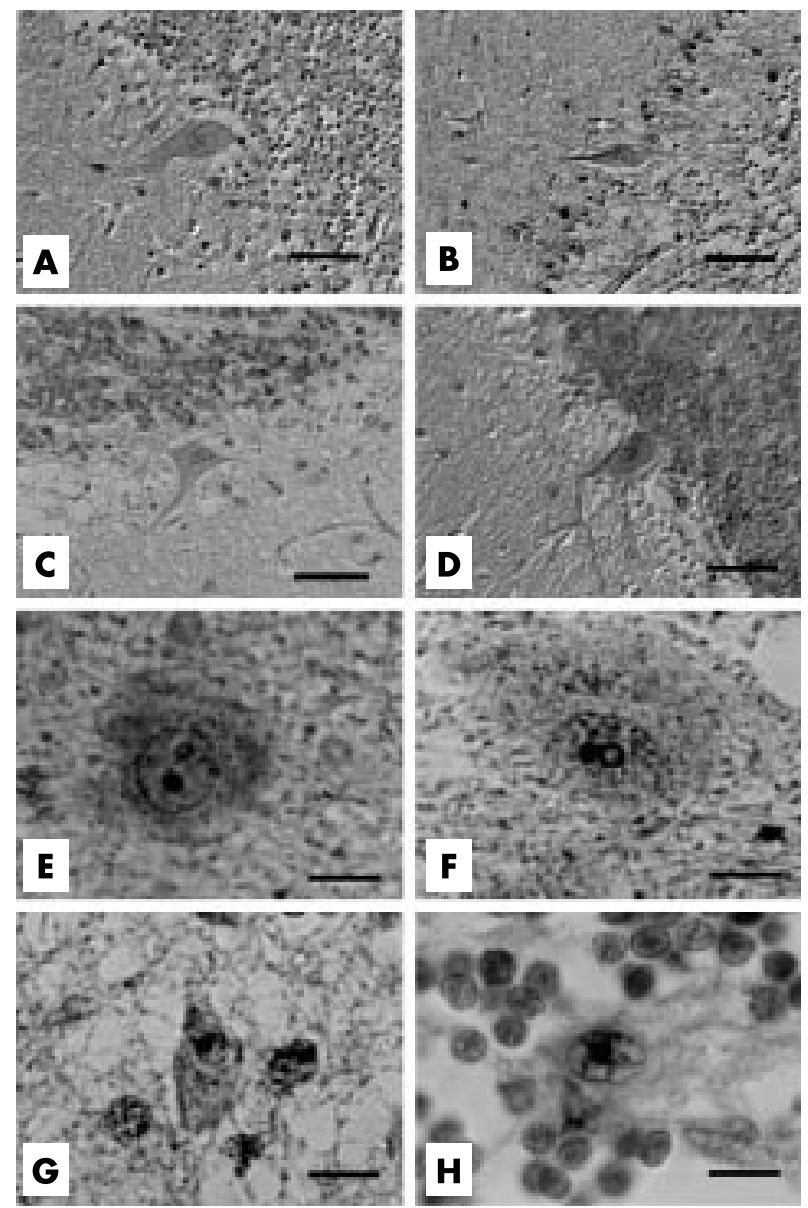

Figure 1 Ubiquitin-like immunoreactivity $(A-G)$ in cerebellar cortex of SCA1 (A), SCA2 (B), SCA3 (C), and DRPLA (D). Neither aggregates nor inclusions were present in nuclei of Purkinje cells in any of cases examined (bars $=100 \mu \mathrm{m}$; A-D: the same

magnification). Nls were occasionally found in dentate neurons in SCA3 (E), DRPLA (F) and rarely found in Golgi cells, as seen in SCAl (G). These Nls were also immuostained with the antibody against expanded polyglutamine (1C2), as seen in Golgi cells in SCAl (H) (bars=10 $\mu \mathrm{m}$; E-H: the same magnification).

Table 1 Number of Pukinje cells in hereditary ataxias

\begin{tabular}{llll}
\hline & Cases $(\mathbf{n})$ & Range & Average \\
\hline SCA1 & 3 & $310-600$ & 420 \\
SCA2 & 3 & $140-160$ & 150 \\
SCA3 & 5 & $1290-3720$ & 2320 \\
DRPLA & 4 & $1600-4560$ & 3130 \\
\hline
\end{tabular}

NIs in any of the remaining Pj cells irrespective of the disease examined.

\section{DISCUSSION}

Cerebellar ataxia is one of the important clinical features of SCA1, SCA2, SCA3, and DRPLA, all exhibit cerebellar degeneration of different degree. Cerebellar cortex is severely affected in SCA2 and SCAl, while relative preservation of $\mathrm{Pj}$ cells is a morphological feature of SCA3 and DRPLA. ${ }^{8}$ Different reports, including ours, confirmed the absence of NIs in $\mathrm{Pj}$ cells of SCA2 ${ }^{910}$ and DRPLA ${ }^{11}$ brains. In this study, we tried to expand this observation by comparing a variety of cases with different degree of cerebellar degeneration associated in common with pathological expansion of CAG repeat. Although several animal models were successfully generated to reproduce NI formation and neurodegeneration in the cerebellar cortex, ${ }^{12}$ our in vivo observation based on a series of human necropsied cases, which includes a variety of hereditary ataxias, failed to identify NIs in any of Pj cells. Because some NIs were identified in dentate neurones or Golgi cells on the same ubiquitin immunolabelled preparations, the absence of NIs in Pj cells was not attributable to a technical failure. Furthermore, we confirmed the absence of NIs in Pj cells by immunohistochemical examination with $1 \mathrm{C} 2$, which labels the pathological moiety of abnormal gene product found in NIs.

At least two conflicting interpretations are possible to explain this consistent absence of NIs in Pj cells. Pj cells containing NIs may be more susceptible to neuronal degeneration and degenerate rapidly, leaving only those not containing NIs. If Pj cells develop NIs before degeneration, however, some NIs should have appeared at least once during the degenerative process. The consistent absence of NIs in Pj cells with various severity of degeneration linked to different genetic abnormalities, therefore, is against this hypothesis. On the other hand, accumulating evidence claimed that neurodegeneration and NI formation were not necessarily correlated ${ }^{13}$ or even inversely correlated ${ }^{7}$ with each other, consistent with a possible protective role of NIs against neuronal degeneration. Indeed, inactivation of ubiquitin-proteasome cascade was reported to accelerate cell death and attenuate formation of NIs induced in cultured cell lines transfected with expanded CAG tagged to ataxin- $1 .{ }^{6}$ Inversely, inactivation of caspases, which counteracted cell death, was reported to promote NI formation. ${ }^{7}$ This inverse relation between NI formation and cell death has been verified also in necropsied brains with Huntington's disease ${ }^{4}$ or with NIHID, ${ }^{5}$ which exhibit similar ubiquitin-immunopositive inclusions. It supposes, therefore, an intrinsic cellular mechanism that links cell death and NI formation in opposite directions, although both of them are triggered by the expansion of CAG/polyglutamine repeat. As shown in this study, Pj cells without NIs could, therefore, be more vulnerable if NI formation is linked to a mechanism counteracting a cascade that accelerates neuronal death.

It remains to be proved, however, why $\mathrm{Pj}$ cells hardly develop NIs in human brains. Because targeted expression of a mutated gene (for example, ataxin-1) to Pj cells induces NIs in murine brains, ${ }^{14}$ mechanisms involved after the expression of the mutated gene may be different in human Pj cells. Otherwise, smaller repeat size in the cerebellum, a common phenomenon in several CAG/polyglutamine repeat expansion disorders known as "somatic mosaicism", may locally influence the phenotype by modifying cell death and NI formation. Recent reports demonstrated, however, that transcribed repeat size in Pj cells of DRPLA brains was longer than that in cerebellar granule cells, ${ }^{15}{ }^{16}$ suggesting that the absence of NI in Pj cells is not related to shorter repeat size. Anyway, this study demonstrated that Pj cells, more or less affected in these hereditary ataxias, were uniformly characterised by the paradoxical absence of NIs in necropsied human brains with different CAG/polyglutamine expansion disorders with ataxia. If NI formation is linked to a protective mechanism against cell death, its complete absence renders Pj cells more susceptible to cell death. Further studies are required to clarify cellular and molecular mechanism to explain these particular characteristics of human Pj cells seen commonly in these hereditary ataxias. It may provide clues to understanding a common mechanistic link, which involves cell death and NI formation both triggered by CAG repeat expansion.

\section{ACKNOWLEDGEMENTS}

This work was supported by a grant from the Research Committee for Ataxic Diseases from the Ministry of Health and Welfare, Japan (KI) 
and by grants from the Ministry of Health and Welfare, Japan $(10223570, \mathrm{TU})$ and from the Japan Health Sciences Foundation (22088, TU). We would like to thank Ms A Nakamura (Department of Neuropathology, Tokyo Metropolitan Institute for Neuroscience) and Ms K Yamaoka (Department of Pathology, Kanagawa Rehabilitation Centre) who provided excellent technical support for pathological examinations.

\section{Addendum}

A recent study on knocked-in mice with SCAl gene flanking expanded CAG repeat demonstrated an extreme scarcity of intranuclear inclusions in degenerated cerebellar Purkinje cells. This corroborated our findings on human necropsied brains (Neuron 2002;34:90519).

\section{Authors' affiliations}

S Koyano, Y Kuroiwa, Department of Neurology, Yokohama City University, Yokohama, Japan

K Iwabuchi, Department of Neurology and Psychiatry, Kanagawa

Rehabilitation Centre, Atsugi, Japan

S Yagishita, Department of Pathology, Kanagawa Rehabilitation Centre, Kanagawa

S Koyano, T Uchihara, Department of Neuropathology, Tokyo

Metropolitan Institute for Neuroscience, Tokyo, Japan

Competing interests: none declared.

Correspondence to: $\operatorname{Dr} T$ Uchihara, Department of Neuropathology, Tokyo Metropolitan Institute for Neuroscience, 2-6 Musashi-dai,

Fuchu-shi, Tokyo 8526, Japan; uchihara@tmin.ac.jp

Received 1 March 2002

In revised form 5 June 2002

Accepted 6 June 2002

\section{REFERENCES}

1 Zoghbi HY, Orr HT. Glutamine repeats and neurodegeneration. Annu Rev Neurosci 2000;23:217-47

2 Holmes SE, O'Hearn E, Mclnnis MG, et al. Expansion of a novel CAG trinucleotide repeat in the $5^{\prime}$ region of PPP2R2B is associated with SCA12. Nat Genet 1999;23:391-2.
3 Nakamura K, Jeong S-Y, Uchihara T, et al. SCA 17, a novel autosomal dominant cerebellar ataxia caused by an expanded polyglutamine in TATA-binding protein. Hum Mol Genet 2001;10:1441-8.

4 Kuemmerle S, Gutekunst C-A, Klein AM, et al. Huntingtin aggregates may not predict neuronal death in Huntington's disease. Ann Neurol 1999;46:842-9.

5 Takahashi J, Tanaka J, Arai K, et al. Recruitment of nonexpanded polyglutamine proteins to intranuclear aggregates in neuronal intranuclear hyaline inclusion disease. I Neuropathol Exp Neurol $2001 ; 60: 369-76$

6 Cummings CJ, Reinstein E, Sun Y, et al. Mutation of the E6-AP ubiquitin ligase reduces nuclear inclusion frequency while accelerating polyglutamine-induced pathology in SCA1 mice. Cell 1999;24:879-92.

7 Saudou F, Finkbeiner S, Devys D, et al. Huntingtin acts in the nucleus to induce apoptosis but death does not correlate with the formation of intranuclear inclusions. Cell 1998;95:55-66.

8 Iwabuchi K, Tsuchiya K, Uchihara T, et al. Autosomal dominant spinocerebellar degenerations. Clinical, pathological and genetic correlations. Rev Neurol (Paris) 1999; 155:255-70.

9 Huynh DP, DelBigio MR, Ho DH, et al. Expression of ataxins-2 in brains from normal individuals and patients with Alzheimer's disease and spinocerebellar ataxia 2. Ann Neurol 1999;45:232-41.

10 Koyano S, Uchihara T, Fujigasaki H, et al. Neuronal intranuclear inclusions in spinocerebellar ataxia type 2: triple-labeling immunofluorescent study. Neurosci Lett 1999;273:117-20.

11 Hayashi Y, Kakita A, Yamada M, et al. Hereditary dentatorubral-pallidoluysian atrophy : detection of widespread ubiquitinated neuronal and glial intranuclear inclusions in the brain. Acta Neuropathol 1998;96:547-52.

12 Skinner PJ, Koshy BT, Cummings CJ, et al. Ataxin-1 with an expanded glutamine tract alters nuclear matrix-associated structures. Nature 1997;389:971-4.

13 Huynh DP, Figueroa K, Hoang N, et al. Nuclear localization or inclusion body formation of ataxin-2 are not necessary for SCA2 pathogenesis in mouse or human. Nat Genet 2000;26:44-50.

14 Klement IA, Skinner PJ, Kaytor MD, et al. Ataxin-1 nuclear localization and aggregation: role in polyglutamine-induced disease in SCA 1 transgenic mice. Cell 1998;95:41-53.

15 Hashida H, Goto J, Suzuki T, et al. Single cell analysis of CAG repeat in brains of dentatorubral-pallidoluysian atrophy. J Neurol Sci 2001;190:87-93.

16 Watanabe H, Tanaka F, Doyu M, et al. Differential somatic CAG repea instability in variable brain cell lineage in dentatorubral pallidoluysian atrophy (DRPLA): a laser-captured microdissection (LCM)-based analysis. Hum Gen 2000;107:452-7. 